\section{Part 3. Advanced cardiac life support: 2015 Korean Guidelines for Cardiopulmonary Resuscitation}

\author{
Mi Jin Lee', Tai Ho Rho ${ }^{2}$, Hyun Kim ${ }^{3}$, Gu Hyun Kang ${ }^{4}$, June Soo Kim ${ }^{5}$, \\ Sang Gyun Rho ${ }^{6}$, Hyun Kyung Park ${ }^{7}$, Dong Jin $\mathrm{Oh}^{8}$, Seil $\mathrm{Oh}^{9}$, Jin Wi ${ }^{10}$, \\ Sangmo Je ${ }^{11}$, Sung Phil Chung ${ }^{12}$, Sung Oh Hwang ${ }^{3}$
}

\begin{abstract}
'Department of Emergency Medicine, Kyungpook National University College of Medicine, Daegu, Korea ${ }^{2}$ Division of Cardiology, Department of Internal Medicine, The Catholic University of Korea College of Medicine, Seoul, Korea

${ }^{3}$ Department of Emergency Medicine, Yonsei University Wonju College of Medicine, Wonju, Korea ${ }^{4}$ Department of Emergency Medicine, Hallym University College of Medicine, Seoul, Korea ${ }^{5}$ Department of Internal Medicine, Sungkyunkwan University School of Medicine, Seoul, Korea ${ }^{6}$ Department of Emergency Medical Services, Sunmoon University, Asan, Korea

${ }^{7}$ Department of Emergency Medicine, Kyung Hee University College of Medicine, Seoul, Korea ${ }^{8}$ Department of Internal Medicine, Hallym University College of Medicine, Seoul, Korea ${ }^{9}$ Department of Internal Medicine, Seoul National University College of Medicine, Seoul, Korea ${ }^{10}$ Department of Internal Medicine, Yonsei University College of Medicine, Seoul, Korea ${ }^{11}$ Department of Emergency Medicine, CHA University College of Medicine, Seongnam, Korea ${ }^{12}$ Department of Emergency Medicine, Yonsei University College of Medicine, Seoul, Korea
\end{abstract}

\section{BACKGROUND OF 2015 GUIDELINES FOR ADULT ADVANCED LIFE SUPPORT}

The chain of survival suggested in the 2015 Korean cardiopulmonary resuscitation (CPR) guidelines consists of the five links of prevention and early detection of cardiac arrest, early access, early cardiopulmonary resuscitation, early defibrillation, and effective advanced life support (ALS) and post-cardiac arrest care. ALS, which constitutes the last link, should immediately follow the basic life support (BLS) if the required manpower, equipment, and drugs are available. ALS includes the following elements: quick recognition of cardiac arrest and activation of the resuscitation team; immediate CPR, manual defibrillation, and pharmacological therapy; and advanced airway management and monitoring of physiological parameters. ${ }^{1}$ Comprehensive post-cardiac arrest care, which begins immediately after recovery of spontaneous circulation, is also included in ALS. Improvements in the survival and neurologic outcomes in patients with cardiac arrest can be expected only when BLS and ALS are provided in combination. In case of in-hospital cardiac arrest, effective BLS begins with the first responder quickly confirming cardiac arrest, immediately calling for help, asking for manual defibrillator while calling the resuscitation team in the hospital, and conducting a high-quality CPR. The most important principle in cardiac arrest treatment through ALS is that it should be based on effective BLS. When ventricular fibrillation (VF) or pulseless ventricular tachycardia (VT) occurs, the survival rate can be increased with immediate CPR and early defibrillation conducted by the witness. Advanced airway management, mechanical chest compression devices, and extracorporeal circulation devices increase the shortterm survival rate including the return of spontaneous circulation (ROSC) and survival admission. $^{2-7}$ However, the evidence supporting that these improve the survival or neurologic out-
eISSN: 2383-4625

Received: 16 February 2016

Revised: 19 March 2016

Accepted: 19 March 2016

Correspondence to: Tai Ho Rho Department of Internal Medicine, St. Paul's Hospital, The Catholic University of Korea College of Medicine, 180 Wangsan-ro, Dongdaemun-gu, Seoul 02559, Korea

E-mail: tairho@catholic.ac.kr

Hyun Kim

Department of Emergency Medicine, Yonsei University Wonju College of Medicine, 20 Ilsan-ro, Wonju 26426, Korea

E-mail:khyun@yonsei.ac.kr

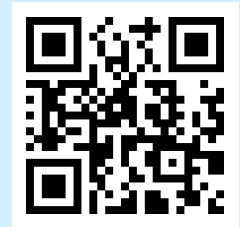

How to cite this article: Lee MJ, Rho TH, Kim H, Kang GH, Kim JS, Rho SG, Park HK, Oh DJ, Oh S, Wi J, Je S, Chung SP, Hwang SO. Part 3. Advanced cardiac life support: 2015 Korean Guidelines for Cardiopulmonary Resuscitation. Clin Exp Emerg Med 2016;3(S):S17-S26.

This is an Open Access article distributed under the terms of the Creative Commons Attribution Non-Commercial License (http:// creativecommons.org/licenses/by-nc/3.0/). 
comes at hospital discharge is still insufficient. Long-term survival of patients who recover their spontaneous circulation can be improved when combined post-cardiac arrest treatment, which includes targeted temperature management and percutaneous coronary intervention, is provided in association with high-quality BLS and effective ALS. ${ }^{8-15}$ The incidence rate of in-hospital cardiac arrest can be decreased by early recognition of patients with deteriorating condition indicating an impending cardiac arrest and responding immediately. ${ }^{16}$

The major issues of ALS described in the 2015 Korean CPR guidelines maintained the basic principles suggested in the 2011 Korean CPR guidelines. The 2015 guidelines continue to emphasize on the importance of immediate and effective CPR (chest compression with adequate depth and rate, sufficient relaxation, minimal interruption of chest compression, and prevention of hyperventilation) in patients who experience cardiac arrest. ${ }^{17-21}$ During CPR, the ALS provider uses available monitoring devices, such as end-tidal carbon dioxide $\left(\mathrm{ETCO}_{2}\right)$ monitoring devices and pulse oximeter, supplies oxygen via advanced airway, and conducts manual defibrillation. Furthermore, during ALS, the provider finds the reversible causes of cardiac arrest and corrects them if possible. As ways of advanced airway management, the use of supraglottic airway devices (e.g., laryngeal mask airway, laryngeal tube, i-gel) is recommended in addition to endotracheal intubation. ${ }^{22-24}$ However, the guideline emphasizes that chest compression should not be delayed due to advanced airway management conducted during CPR.

\section{HIGHLIGHTS OF 2015 GUIDELINES FOR ADULT ALS}

The key issues and the major highlights of the 2015 Korean guidelines for ALS include the following. Monitoring physiologic parameters during CPR provides meaningful information about the patient's condition and response to CPR. These parameters can be monitored continuously, without interrupting chest compression. The use of quantitative waveform capnography for monitoring of the partial pressure of $\mathrm{ETCO}_{2}$ is recommended after advanced airway management. Capnography is useful for evaluating the effectiveness of chest compression and the possibility of the ROSC during CPR. ${ }^{25,26}$ Low ETCO 2 in intubated patients after 20 minutes of CPR is associated with poor chances for ROSC and survival. ${ }^{26,27}$ When quantitative capnography is used after endotracheal intubation, the healthcare providers can confirm an intratracheal placement of endotracheal tube..$^{28,29}$

With regard to vasopressors for cardiac arrest treatment, the combination of vasopressin and epinephrine offers no advantage over the standard-dose epinephrine. Vasopressin has been removed from the adult out-of-hospital cardiac arrest algorithm. However, as per the 2015 Korean CPR guidelines for ALS, vasopressin may provide some benefit when bundled with epinephrine and steroids in treating in-hospital cardiac arrest. ${ }^{30,31}$

Although mechanical chest compression devices have been developed and are currently being used in cardiac arrest patients, the use of such devices as a routine alternative to the conventional CPR is not recommended. Manual chest compression remains the standard care for cardiac arrest; however, in certain circumstances (e.g., in case of limited manpower or prolonged $\mathrm{CPR}$, when in a moving ambulance or helicopter, in the angiography room, or during preparation of extracorporeal CPR [ECPR]), the use of mechanical chest compression devices can be considered as a reasonable alternative to high-quality chest compression. ${ }^{5,32,33}$ ECPR is a complex intervention that requires a highly trained team, specialized equipment, and multidisciplinary support. Only for selected patients who have a cardiac arrest and for whom the suspected etiology of cardiac arrest is potentially reversible, ECPR may be considered an alternative to conventional $\mathrm{CPR}^{6,7}$

\section{CARDIAC ARREST ALGORITHM IN ADULT}

Because the necessity of defibrillation and the drugs administered for treatment of cardiac arrest differ depending on electrocardiography (ECG) rhythms, understanding of the entire algorithm and initial ECG rhythms is essential for effective ALS. Cardiac rhythms during cardiac arrest include shockable rhythms (VF and pulseless VT) and non-shockable rhythms (asystole and pulseless electrical activity [PEA]). BLS and early defibrillation, rather than advanced airway management or drug administration, are the most important and preferentially provided treatment in cardiac arrest. Few drugs used in the treatment of cardiac arrest are supported by evidence. After starting CPR and attempting defibrillation, rescuers can establish intravenous (IV) access, considering drug administration, and then insert an advanced airway.

As in the 2011 guideline, the 2015 guideline combines the treatment processes of cardiac arrest into a universal algorithm; this also corresponds to the "megacode concept" used in ALS education. In other words, because changes in ECG rhythms may occur during ALS provided for cardiac arrest, a combined algorithm, rather than separate algorithms for each ECG rhythm, can be more effectively used in clinical field. For instance, the scenario in which "VF occurs while treating asystole, and spontaneous circulation is recovered after defibrillation," could occur in prac- 
tice; in such cases, if VF is observed while treating asystole, the treatment direction should change to treat VF first.

The treatment of cardiac arrest starts with BLS. BLS includes the recognition of the patient, confirmation of cardiac arrest, call for help, and immediate beginning CPR. Regardless of the ECG rhythm, push hard and fast and release completely in chest compression. Moreover, to minimize the fatigue of the chest compressors, the CPR team leader should switch the roles with the compressor approximately every 2 minutes (or after 5 cycles of compressions and ventilations at a ratio of 30:2). Healthcare providers should interrupt chest compressions as infrequently as possible and try to limit interruptions to no longer than $10 \mathrm{sec}-$ onds except for specific interventions such as use of a defibrillator or analyzing the ECG rhythms through monitors, confirmation of pulse for confirmation of spontaneous circulation (when perfusing rhythm appears on ECG), and insertion of an advanced airway. As ways to evaluate the effectiveness and quality of CPR, there are a number of methods and emerging technologies to monitor the patient during CPR and potentially help guide ALS interventions (e.g., monitoring of $\mathrm{ETCO}_{2}$ partial pressure, diastolic arterial pressure, and central venous oxygen saturation). When an advanced airway is not in placement, chest compression and rescue ventilation should to be conducted in a 30:2 ratio; when the advanced airway is secured, chest compression should be con- ducted at 100-120 times/min while ventilation should be conducted once in 6 seconds (10 times/min). ${ }^{21}$ Hyperventilation should be avoided. Manual defibrillators, rather than automated defibrillators, are used in ALS to confirm ECG rhythms and to defibrillate.

Although administration of drugs before conducting advanced airway management is recommended, depending on the team members' skills as well as the ongoing experience in inserting the airway and verifying proper position with minimal interruption of chest compression, drug administration and advanced airway management can be conducted simultaneously. In patients with an initial shockable rhythm, administration of epinephrine (IV or intraosseous [IO] injection) immediately after the first shock is recommended. In cases of asystole or PEA (non-shockable rhythms), early administration of epinephrine can increase the ROSC, survival to hospital discharge, and neurologically intact survival. ${ }^{34-36}$ This section discusses in detail the general management of patients experiencing cardiac arrest and provides an overview of the ALS Cardiac Arrest Algorithm (Fig. 1 and Table 1).

\section{Shockable rhythms (VF/pulseless VT)}

Because VF and pulseless VT can be treated with defibrillation, they are referred to as shockable rhythms. The most important treatment for VF or pulseless VT is immediate bystander CPR and

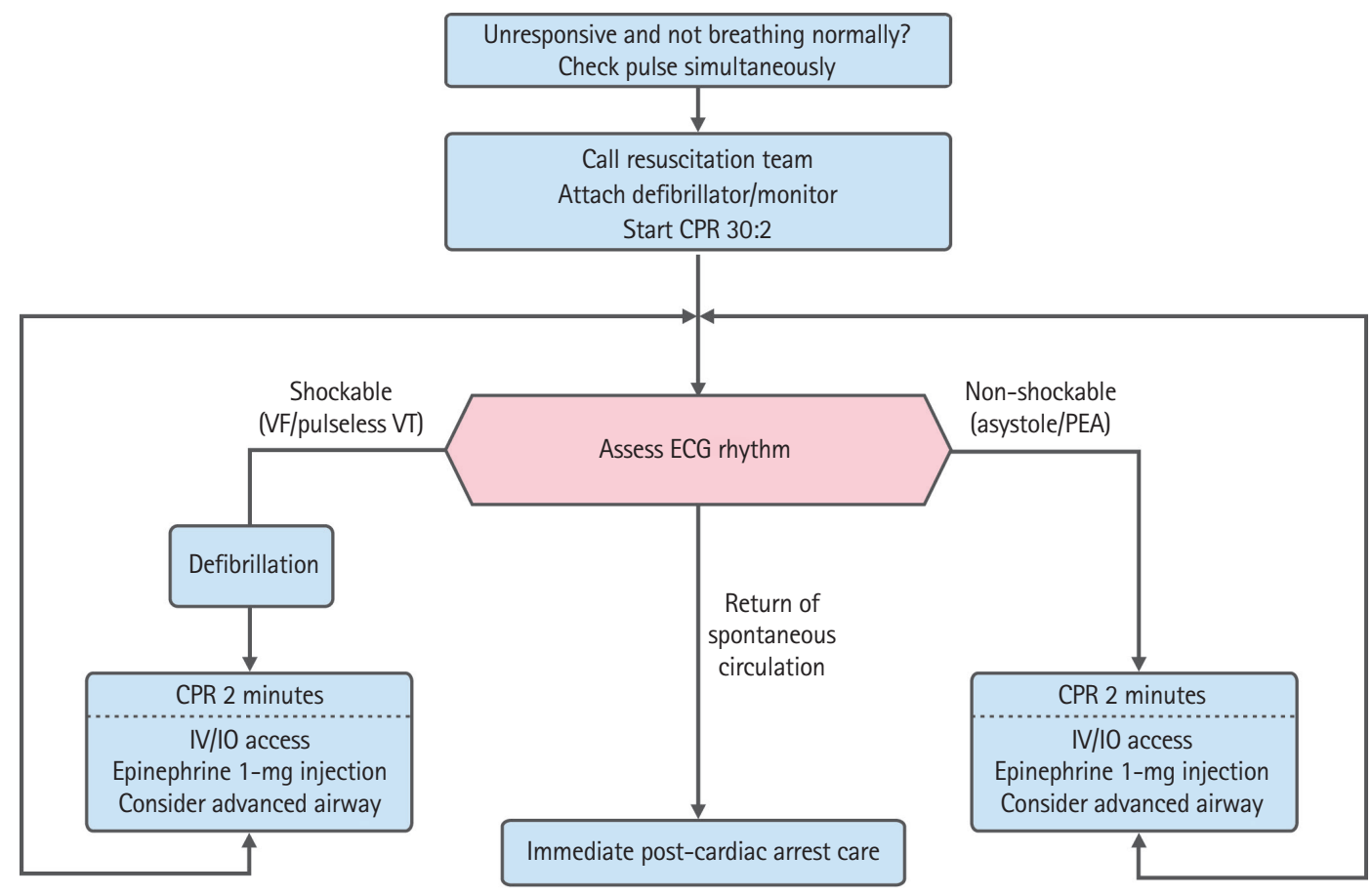

Fig. 1. Adult advanced life support algorithm. CPR, cardiopulmonary resuscitation; VF, ventricular fibrillation; VT, ventricular tachycardia; ECG, electrocardiography; PEA, pulseless electrical activity; IV, intravenous; IO, intraosseous. 
Table 1. Reference table of the adult ALS algorithm

\begin{tabular}{|c|c|}
\hline Core ALS concepts & Details \\
\hline Assess ECG rhythm & Rotate a compressor and analyze rhythm every 2 minutes \\
\hline Defibrillation & $\begin{array}{l}\text { Biphasic: } \\
\text { Initial dose } 120 \text { to } 200 \mathrm{~J} \text { (manufacturer recommendation) } \\
\text { Refractory VF/pulseless VT: escalating doses } \\
\text { Monophasic: } 360 \mathrm{~J}\end{array}$ \\
\hline Chest compression & $\begin{array}{l}\text { Push hard }(5 \mathrm{~cm}) \text { and fast }(100-120 / \mathrm{min}) \\
\text { Ensure high quality chest compressions } \\
\text { Start compression within } 5 \text { seconds after defibrillation } \\
\text { Use waveform capnography (achieve } \mathrm{ETCO}_{2}>10 \mathrm{mmHg} \text { after endotracheal intubation or } 20 \text { minutes of CPR) }\end{array}$ \\
\hline $\begin{array}{l}\text { Advanced airway management } \\
\text { and ventilation }\end{array}$ & $\begin{array}{l}\text { Keep bag-valve-mask ventilation until advanced airway in place } \\
\text { Perform endotracheal intubation or use a supraglottic airway } \\
\text { Give } 1 \text { breath every } 6 \text { seconds (10 breaths/min) with continuous chest compressions } \\
\text { Avoid hyperventilation }\end{array}$ \\
\hline \multicolumn{2}{|l|}{ Drug administration (IV/IO) } \\
\hline Refractory VF/pulseless VT & $\begin{array}{l}\text { Amiodarone: } 300 \mathrm{mg} \text { bolus (first dose), } 150 \mathrm{mg} \text { (second dose) } \\
\text { Lidocaine (as an alternative if amiodarone is not available): } 1-1.5 \mathrm{mg} / \mathrm{kg} \text { (first dose), } 0.5-0.75 \mathrm{mg} / \mathrm{kg} \text { (second dose) }\end{array}$ \\
\hline Treat reversible causes & $\begin{array}{l}\text { Hypovolemia, hypoxia, hydrogen ion (acidosis), hypothermia, hypo/hyperkalemia, tension pneumothorax, toxins, } \\
\text { tamponade-cardiac, thrombosis-coronary or pulmonary }\end{array}$ \\
\hline Consider additional CPR modalities & $\begin{array}{l}\text { Ultrasound imaging } \\
\text { Mechanical chest compressions } \\
\text { Extracorporeal CPR }\end{array}$ \\
\hline
\end{tabular}

ALS, advanced life support; ECG, electrocardiography; $\mathrm{ETCO}_{2}$, end-tidal carbon dioxide; CPR, cardiopulmonary resuscitation; IV, intravenous; IO, intraosseous; VF, ventricular fibrillation; VT, ventricular tachycardia.

defibrillation as soon as possible. When VF is found on ECG monitor during CPR, providers should deliver one shock (biphasic defibrillator, 120-200J; monophasic defibrillator, 360J) and then resume CPR immediately for 2 minutes, beginning with chest compressions without pulse check or ECG rhythm confirmation. When the defibrillation energy dose recommended by the manufacturer is not known, defibrillation should be conducted at 200J when a biphasic defibrillator is used. During chest compression, an IV or I0 route for drug administration should be established. ECG rhythms should be checked after two minutes of CPR. If VF persists, the second and subsequent energy doses should be equivalent, and higher doses may be considered. ${ }^{37,38}$ The entire process of manual defibrillation should be achievable in less than a 5 -second interruption to chest compression. ${ }^{39,40}$

During chest compression, epinephrine should be injected, and bolus IV fluids need to be pushed through the site of injection immediately. Meanwhile, another rescuer should perform advanced airway management. After 2 minutes of chest compressions, analyze the rhythm and deliver another shock immediately if indicated. Although $1 \mathrm{mg}$ of epinephrine should be administered every 3 to 5 minutes in principle, it can be administered every two CPR cycles (4 minutes when rescuers take turns in conducting chest compression every 2 minutes). When VF/pulseless
VT persists after 2 to 3 shocks, consider administering an antiarrhythmic such as amiodarone. Three hundred milligram of amiodarone is recommended as the first dose; when VF/pulseless VT persists, additional $150 \mathrm{mg}$ can be administered after 4 minutes from initial administration. If amiodarone is not available, lidocaine may be considered as an alternative. Although the previous 2011 guidelines suggested consideration of magnesium administration for refractory $V F$, the new guidelines do not recommend routine use of magnesium any more, as it does not result in any improvement in survival prognosis. ${ }^{41}$

If the ECG rhythms change to asystole or PEA while treating for VF/pulseless $V T$, the treatment should proceed according to the algorithm for non-shockable rhythms.

\section{Non-shockable rhythms (PEA and asystole)}

Asystole refers to ventricular asystole; PEA is a heterogeneous group of pulseless rhythms that includes pseudo-electromechanical dissociation, idioventricular rhythms, ventricular escape rhythms, and bradyasystolic rhythms. As asystole and PEA do not require defibrillation during $C P R$, they are referred to as "nonshockable rhythms." PEA is often caused by reversible conditions and can be treated if those conditions are identified and corrected. Typical examples of such reversible causes are often referred 
to as the "5Hs and 5Ts": hypovolemic shock, hypoxia, hyperkalemia or hypokalemia, hydrogen ions (metabolic acidosis), hypothermia (the 5Hs), thromboembolism of the pulmonary artery (pulmonary embolism), thrombosis of the coronary artery (myocardial infarction), tension pneumothorax, cardiac tamponade, and toxins (the 5Ts). Survival from cardiac arrest with asystole is poor. Of note, the hope for resuscitation is to identify and treat a reversible cause of non-shockable rhythms.

If the ECG rhythm is non-shockable, chest compression should be immediately performed for 2 minutes. If the QRS complex can be confirmed in PEA, the pulse should be quickly checked. If there is no pulse, CPR should be conducted for 2 minutes while the route (IV or IO) for drug administration is established. Epinephrine should be administered every 3 to 5 minutes or every two CPR cycles (4 minutes). After 2 minutes of chest compression, rhythms should be analyzed. If asystole or PEA persists, chest compression should be continued, and then advanced airway management should be conducted. Atropine is no longer used in treatment of asystole.

\section{Immediate post-cardiac arrest treatment}

Post-cardiac arrest care is offered to patients whose spontaneous circulation has recovered as a result of ALS care. Integrated postcardiac arrest treatment, which includes hemodynamic stabilization, adequate ventilation and controlling of blood oxygen saturation, targeted temperature management, and percutaneous coronary intervention for acute coronary syndrome, should be provided immediately after the recovery of spontaneous circulation.

\section{In-hospital resuscitation}

For all in-hospital cardiac arrests, cardiopulmonary arrest should be recognized immediately and CPR should be started immediately; if indicated, defibrillation is attempted as soon as possible and certainly within 3 minutes. The importance of "prevention and early detection of cardiac arrest," which is the first link suggested in the 2015 CPR guidelines, is emphasized not only in outof-hospital cardiac arrest, but also in in-hospital cardiac arrest. The resuscitation team may take the form of a traditional CPR team, which is called only when cardiac arrest is detected. Alternatively, hospitals may have strategies to engage rapid response teams or medical emergency teams to recognize patients at risk of cardiac arrest before cardiac arrest occurs. ${ }^{16,42,43}$ Contrary to out-of-hospital cardiac arrest, vasopressin may provide some benefit when bundled with epinephrine and steroid in treating in-hospital cardiac arrests. ${ }^{30,31}$

\section{MONITORING DURING ALS}

Monitoring both provider performance and patient physiologic parameters during CPR is essential to optimizing CPR quality and feedback on the effectiveness of chest compression. ${ }^{44} \mathrm{ETCO}_{2}$ partial pressure, coronary perfusion pressure, and central venous oxygen saturation can be used as physiological monitoring indices during ALS. It is recommended to use quantitative waveform capnography in intubated patients to monitor CPR quality, optimize chest compressions, detect ROSC during chest compression or when rhythm check reveals an organized rhythm, and confirmation of the location of endotracheal intubation. ${ }^{25,26,29}$ Although placement of invasive monitors during CPR is not generally warranted, physiologic parameters such as arterial pressures and central venous oxygen saturation, when available, may also be helpful in optimizing CPR and detecting ROSC.

The main determinant of $\mathrm{ETCO}_{2}$ during $\mathrm{CPR}$ is perfusion to the lungs. Persistently low $\mathrm{ETCO}_{2}$ values $<10 \mathrm{mmHg}$ during $\mathrm{CPR}$ in intubated patients suggest that ROSC is unlikely. Monitoring the $\mathrm{ETCO}_{2}$ during ALS can help in maintaining high-quality chest compression and detecting compressor fatigue. ${ }^{45,46}$ If the $\mathrm{ETCO}_{2}$ partial pressure is measured to be less than $10 \mathrm{mmHg}$, the quality of ALS should be improved by increasing performance or changing a compressor. If $\mathrm{ETCO}_{2}$ abruptly increases to a normal value of 35 to $40 \mathrm{mmHg}$, it is reasonable to consider this an indicator of ROSC. Continued insufficient $\mathrm{ETCO}_{2}(<10 \mathrm{mmHg})$ after intubation and 20 minutes after the initial resuscitation is associated with extremely poor chances for ROSC and survival outcomes. ${ }^{26,27,47}$ In non-intubated patients (those with bag-mask ventilation or supraglottic airway), $\mathrm{ETCO}_{2}$ may not consistently reflect the true value, making the measurement less reliable as a prognostic tool.' However, the measurement of $\mathrm{ETCO}_{2}$ partial pressure should not be used as the single index to monitor the quality of ALS or to predict the recovery of spontaneous circulation.

\section{MEDICATIONS FOR CARDIAC ARREST}

High-quality CPR and rapid defibrillation are most important in cardiac arrest, and drug administration is considered next. After starting CPR and conducting defibrillation, IV or I0 access has been established for drug administration. The establishment of peripheral access is preferred because it does not require interruption of chest compression. In cases of shockable rhythms, defibrillation should be conducted first, and epinephrine administration follows afterward; in cases of non-shockable rhythms, drugs should be administered immediately and early once chest compression begins and once the IV route is achieved. ${ }^{36}$ 


\section{Epinephrine}

Epinephrine is the universal vasopressor used in cardiac arrest treatment. Epinephrine stimulates adrenergic receptors, produces vasoconstriction, increases blood pressure and heart rate, and improves perfusion pressure to the brain and heart. Based on improvements in short-term prognosis resulting from epinephrine as compared to placebo, administration of standard dose of epinephrine to cardiac arrest patients is recommended. However, it is not recommended to add vasopressin to standard-dose epinephrine. High-dose epinephrine is not recommended for routine use in cardiac arrest. ${ }^{48}$ Standard-dose epinephrine $(1 \mathrm{mg}$ every 3 to 5 minutes) is recommended for patients experiencing cardiac arrest. The standard protocol used during CPR for adults is as follows: $1 \mathrm{mg}$ of epinephrine is administered quickly in the form of a 1:1,000 ampule or 1:10,000 prefilled syringe formula every 3 to 5 minutes or every two cycles of chest compression rotation (4 minutes). ${ }^{49,50}$ Either IV or 10 routes can be used. If IV/IO access is delayed or cannot be established, epinephrine may be given by the endotracheal route at a dose of 2 to $2.5 \mathrm{mg}^{51}$

The timing of epinephrine administration during CPR depends on the cardiac arrest rhythm. In cardiac arrest patients with a non-shockable rhythm, early administration of epinephrine is suggested. ${ }^{34}$ Optimal timing of epinephrine cannot be recommended because of insufficient evidence, particularly in relation to defibrillation in cardiac arrest due to a shockable rhythm. In such cases, early defibrillation, rather than the timing of epinephrine administration, should be considered first, and epinephrine is quickly administered after that. ${ }^{34,36}$

\section{Vasopressin}

Vasopressin is a non-adrenergic peripheral vasoconstrictor that increases arterial blood pressure. Vasopressin offers no advantage as a substitute for epinephrine in out-of-hospital cardiac arrest. The use of vasopressin in treatment of out-of-hospital cardiac arrest as an alternative to epinephrine is no longer recommended. However, those healthcare professionals working in systems that already use vasopressin may continue to do so because there is no evidence of harm from using vasopressin when compared to epinephrine. ${ }^{47}$

Considering that vasopressin is currently being used in cases of in-hospital cardiac arrest, the evidence to support changing the existing guideline is also lacking. The effect of vasopressin in cardiac arrest does not differ from that of epinephrine; therefore, 40 units of vasopressin can be administered once through an IV or IO route to replace the first or second epinephrine administration. ${ }^{52}$ Moreover, the "vasopressor bundle" combination therapy, which combines steroids, epinephrine, and vasopressin, is being suggested as a treatment option for in-hospital cardiac arrest. ${ }^{30,31}$

\section{Antiarrhythmic drug}

Although the survival to hospital admission rate was reported to increase in a study that investigated the effectiveness of antiarrhythmic drugs in refractory VF/pulseless VT, no antiarrhythmic drug has yet been shown to increase long-term survival or neurologic outcome after cardiac arrest due to VF/pulseless VT. ${ }^{53}$ Amiodarone, however, has been shown to increase short-term survival to hospital admission when compared with placebo or lidocaine. ${ }^{54}$ Although the use of magnesium was recommended until the 2011 guidelines when hypomagnesemia was suspected in patients with cardiac arrest, the new guidelines do not recommend routine use of magnesium for adult VF/pulseless VT patients. ${ }^{41}$

\section{1) Amiodarone}

IV amiodarone affects sodium, potassium, and calcium channels as well as $\alpha$ - and $\beta$-adrenergic blocking actions. Amiodarone is used to treat refractory VF/pulseless VT that does not respond to defibrillation. The initial dose of $300 \mathrm{mg}$ can be injected intravenously or intraosseously; if there is no response, a second dose of $150 \mathrm{mg}$ amiodarone is administered after two cycles of chest compression rotation (after 4 minutes). ${ }^{55}$

\section{2) Lidocaine}

The short-or long-term effects of lidocaine in refractory VF/pulseless VT that does not respond to defibrillation or repeats after defibrillation have been investigated in only a limited number of studies. ${ }^{56,57}$ Lidocaine can be considered in cases where amiodarone is not available. ${ }^{54}$ The initial dose is 1 to $1.5 \mathrm{mg} / \mathrm{kg} \mathrm{IV} / \mathrm{IO}$. Repeat if indicated at 0.5 to $0.75 \mathrm{mg} / \mathrm{kg}$ over 5 - to 10 -minute intervals to a maximum of $3 \mathrm{mg} / \mathrm{kg}$.

\section{ADDITIONAL CONSIDERATIONS DURING ALS}

\section{Oxygen supply during CPR}

A study on out-of-hospital cardiac arrest analyzed outcome according the arterial oxygen partial pressure during CPR and reported that the survival-to-hospital admission rate increased in the group that received high concentration of oxygen.$^{58}$ When supplementary oxygen is available, it may be reasonable to use the maximal feasible inspiratory concentration during CPR. After the recovery of spontaneous circulation, it is recommended to administer the maximum oxygen concentration that can maintain the arterial oxygen saturation above $94 \%$. 


\section{Factors that predict the prognosis during CPR}

The $\mathrm{ETCO}_{2}$, which reflects the cardiac output induced by chest compression, is the only predicting factor currently being used during CPR. Survival prognosis was found to be poor in studies that investigated in-hospital and out-of-hospital cardiac arrest when $\mathrm{ETCO}_{2}$ was maintained at less than $10 \mathrm{mmHg}$ in cardiac arrest patients with endotracheal intubation even 20 minutes after the start of $\mathrm{CPR}^{26,27}$ Hence, an $\mathrm{ETCO}_{2}$ less than $10 \mathrm{mmHg}$ immediately after intubation and 20 minutes after the initial resuscitation is associated with extremely poor chances for ROSC and survival. ${ }^{47}$ The $\mathrm{ETCO}_{2}$ below $10 \mathrm{mmHg}$ that persists even after 20 minutes of appropriate ALS can be used as a criterion, in association with other various factors, to stop ALS. The $\mathrm{ETCO}_{2}$ should not be used alone as an indication to terminate resuscitation efforts. This index cannot be applied in patients that do not have endotracheal intubation. In cases of a simple non-waveform capnography used in pre-hospital care, the measurements can be used to confirm endotracheal intubation. However, these are not recommended for prognostication of survival or as evidence for stopping CPR. Multimodal approach to decision of terminating resuscitation may be required during $C P R$.

\section{Application of ultrasonography during CPR}

The evidence supporting the effects of ultrasonography used during adult CPR is not sufficient. However, the use of ultrasonography may be considered to determine the reversible causes of cardiac arrest only in cases that ultrasonography would not interfere with $C P R .{ }^{59}$ Although no evidence supports that the use of echocardiography improves the prognosis of cardiac arrest patients, it can help in diagnosing the cause of treatable cardiac arrest, including cardiac tamponade, pulmonary thromboembolism, myocardial infarction, and aortic dissection. Moreover, it can also be used as an alternative of $\mathrm{ETCO}_{2}$ capnography to confirm the location of endotracheal intubation during $\mathrm{CPR}^{60}$

\section{The use of mechanical chest compression devices}

Automated mechanical chest compression devices use a piston that compresses the sternum, a band that tightens the thorax, or both to conduct CPR. Since the announcement of the 2005 guidelines, important clinical studies that used mechanical chest compression devices were conducted. As the evidence supporting effectiveness of mechanical chest compression devices over manual CPR is insufficient yet, manual chest compression remains the standard care for cardiac arrest. ${ }^{5,32,33}$ However, mechanical chest compression devices can be used as reasonable alternatives in the following cases: when the conventional chest compression is not possible, when high-quality chest compression cannot be con- ducted due to the prolonged CPR, and when there is a lack of medical personnel required for CPR. In particular, in certain circumstances, such as inside a moving ambulance, inside angiography rooms, during prolonged CPR, and during ECPR, the use of mechanical chest compression devices can be considered as a reasonable alternative to high-quality chest compression.

\section{Extracorporeal CPR}

ECPR maintains artificial circulation using extracorporeal devices in patients with cardiac arrest who do not response to conventional CPR. It is known to exert positive influences on the survival and neurological prognosis of adult cardiac arrest patients; therefore, it can be used in selective circumstances. ${ }^{6,7,61}$ As cannula and devices should be inserted into large arteries and veins while conducting CPR, special equipment and trained members are required. ECPR is a complex intervention that requires a highly trained team, specialized equipment, and multidisciplinary support. Only for selected patients who have a cardiac arrest and for whom the suspected etiology of cardiac arrest is potentially reversible, ECPR may be considered as an alternative to conventional CPR. ${ }^{6,7}$

\section{ACKNOWLEDGMENTS}

Collaborators have been contributed to the review of the scientific evidence and the adjustment of new GRADE methods. The collaborators' are as follows: Eun Jung Park, Ajou University, Suwon, Korea; Hyo Eun Park, Seoul National University, Seoul, Korea; Jeong Hun Lee, Dongguk University, Seoul, Korea; Je Sung You, Yonsei University, Seoul, Korea; Jung Ho Shin, Armed Forces Capital Hospital, Seongnam, Korea; Kyung Woon Jeung, Chonnam National University, Gwangju, Korea; Kyu Seok Kim, Seoul National University, Seoul, Korea; Sang Jin Han, Hallym University, Seoul, Korea; Seong Beom Oh, Dankook University, Cheonan, Korea; Seokran Yeom, Busan National University, Busan, Korea; Seung Min Park, Hallym University, Pyungchon, Korea; Sung Won Jang, Catholic University of Korea, Seoul, Korea; Tae Yong Shin, Bundang Jesaeng General Hospital, Seongnam, Korea; Young Hoon Yoon, Korea University, Seoul, Korea.

\section{REFERENCES}

1. Link MS, Berkow LC, Kudenchuk PJ, et al. Part 7. Adult advanced cardiovascular life support: 2015 American Heart Association Guidelines update for cardiopulmonary resuscitation and emergency cardiovascular care. Circulation 2015;132(18 Suppl 2):S444-64. 
2. Hanif MA, Kaji AH, Niemann JT. Advanced airway management does not improve outcome of out-of-hospital cardiac arrest. Acad Emerg Med 2010;17:926-31.

3. Hasegawa K, Hiraide A, Chang Y, Brown DF. Association of prehospital advanced airway management with neurologic outcome and survival in patients with out-of-hospital cardiac arrest. JAMA 2013;309:257-66.

4. McMullan J, Gerecht R, Bonomo J, et al. Airway management and out-of-hospital cardiac arrest outcome in the CARES registry. Resuscitation 2014;85:617-22.

5. Wik L, Olsen JA, Persse D, et al. Manual vs. integrated automatic load-distributing band CPR with equal survival after out of hospital cardiac arrest: the randomized CIRC trial. Resuscitation 2014;85:741-8.

6. Chen YS, Lin JW, Yu HY, et al. Cardiopulmonary resuscitation with assisted extracorporeal life-support versus conventional cardiopulmonary resuscitation in adults with in-hospital cardiac arrest: an observational study and propensity analysis. Lancet 2008;372:554-61.

7. Sakamoto T, Morimura N, Nagao K, et al. Extracorporeal cardiopulmonary resuscitation versus conventional cardiopulmonary resuscitation in adults with out-of-hospital cardiac arrest: a prospective observational study. Resuscitation 2014; 85:762-8.

8. Hypothermia after Cardiac Arrest Study Group. Mild therapeutic hypothermia to improve the neurologic outcome after cardiac arrest. N Engl J Med 2002;346:549-56.

9. Bernard SA, Gray TW, Buist MD, et al. Treatment of comatose survivors of out-of-hospital cardiac arrest with induced hypothermia. N Engl J Med 2002;346:557-63.

10. Strote JA, Maynard C, Olsufka M, et al. Comparison of role of early (less than six hours) to later (more than six hours) or no cardiac catheterization after resuscitation from out-of-hospital cardiac arrest. Am J Cardiol 2012;109:451-4.

11. Nielsen N, Wetterslev J, Cronberg T, et al. Targeted temperature management at $33^{\circ} \mathrm{C}$ versus $36^{\circ} \mathrm{C}$ after cardiac arrest. $\mathrm{N}$ Engl J Med 2013;369:2197-206.

12. Hollenbeck RD, McPherson JA, Mooney MR, et al. Early cardiac catheterization is associated with improved survival in comatose survivors of cardiac arrest without STEMI. Resuscitation 2014;85:88-95.

13. Grasner JT, Meybohm P, Lefering R, et al. ROSC after cardiac arrest: the RACA score to predict outcome after out-of-hospital cardiac arrest. Eur Heart J 2011;32:1649-56.

14. Bro-Jeppesen J, Kjaergaard J, Wanscher M, et al. Emergency coronary angiography in comatose cardiac arrest patients: do real-life experiences support the guidelines? Eur Heart J
Acute Cardiovasc Care 2012;1:291-301.

15. O'Gara PT, Kushner FG, Ascheim DD, et al. 2013 ACCF/AHA guideline for the management of ST-elevation myocardial infarction: executive summary: a report of the American College of Cardiology Foundation/American Heart Association Task Force on Practice Guidelines. Circulation 2013;127:52955.

16. Maharaj R, Raffaele I, Wendon J. Rapid response systems: a systematic review and meta-analysis. Crit Care 2015;19:254.

17. Stiell IG, Brown SP, Nichol G, et al. What is the optimal chest compression depth during out-of-hospital cardiac arrest resuscitation of adult patients? Circulation 2014;130:1962-70.

18. Idris AH, Guffey D, Pepe PE, et al. Chest compression rates and survival following out-of-hospital cardiac arrest. Crit Care Med 2015;43:840-8.

19. Aufderheide TP, Sigurdsson G, Pirrallo RG, et al. Hyperventilation-induced hypotension during cardiopulmonary resuscitation. Circulation 2004;109:1960-5.

20. O'Neill JF, Deakin CD. Do we hyperventilate cardiac arrest patients? Resuscitation 2007;73:82-5.

21. Abella BS, Alvarado JP, Myklebust $H$, et al. Quality of cardiopulmonary resuscitation during in-hospital cardiac arrest. JAMA 2005;293:305-10.

22. Cook TM, Kelly FE. Time to abandon the 'vintage' laryngeal mask airway and adopt second-generation supraglottic airway devices as first choice. Br J Anaesth 2015;115:497-9.

23. Sunde GA, Brattebo G, Odegarden T, Kjernlie DF, Rodne E, Heltne JK. Laryngeal tube use in out-of-hospital cardiac arrest by paramedics in Norway. Scand J Trauma Resusc Emerg Med 2012;20:84.

24. Duckett J, Fell P, Han K, Kimber C, Taylor C. Introduction of the I-gel supraglottic airway device for prehospital airway management in a UK ambulance service. Emerg Med J 2014;31: 505-7.

25. Pearce AK, Davis DP, Minokadeh A, Sell RE. Initial end-tidal carbon dioxide as a prognostic indicator for inpatient PEA arrest. Resuscitation 2015;92:77-81.

26. Levine RL, Wayne MA, Miller CC. End-tidal carbon dioxide and outcome of out-of-hospital cardiac arrest. N Engl J Med 1997;337:301-6.

27. Ahrens T, Schallom L, Bettorf K, et al. End-tidal carbon dioxide measurements as a prognostic indicator of outcome in cardiac arrest. Am J Crit Care 2001;10:391-8.

28. Silvestri $S$, Ralls GA, Krauss $B$, et al. The effectiveness of outof-hospital use of continuous end-tidal carbon dioxide monitoring on the rate of unrecognized misplaced intubation within a regional emergency medical services system. Ann 
Emerg Med 2005;45:497-503.

29. Grmec S. Comparison of three different methods to confirm tracheal tube placement in emergency intubation. Intensive Care Med 2002;28:701-4.

30. Mentzelopoulos SD, Zakynthinos SG, Tzoufi M, et al. Vasopressin, epinephrine, and corticosteroids for in-hospital cardiac arrest. Arch Intern Med 2009;169:15-24.

31. Mentzelopoulos SD, Malachias S, Chamos C, et al. Vasopres$\sin$, steroids, and epinephrine and neurologically favorable survival after in-hospital cardiac arrest: a randomized clinical trial. JAMA 2013;310:270-9.

32. Rubertsson $S$, Lindgren $E$, Smekal $D$, et al. Mechanical chest compressions and simultaneous defibrillation vs conventional cardiopulmonary resuscitation in out-of-hospital cardiac arrest: the LINC randomized trial. JAMA 2014;311:53-61.

33. Perkins GD, Lall $R$, Quinn $T$, et al. Mechanical versus manual chest compression for out-of-hospital cardiac arrest (PARAMEDIC): a pragmatic, cluster randomised controlled trial. Lancet 2015;385:947-55.

34. Donnino MW, Salciccioli JD, Howell MD, et al. Time to administration of epinephrine and outcome after in-hospital cardiac arrest with non-shockable rhythms: retrospective analysis of large in-hospital data registry. BMJ 2014;348:g3028.

35. Goto $Y$, Maeda T, Goto Y. Effects of prehospital epinephrine during out-of-hospital cardiac arrest with initial non-shockable rhythm: an observational cohort study. Crit Care 2013;17:R188.

36. Koscik C, Pinawin A, McGovern $H$, et al. Rapid epinephrine administration improves early outcomes in out-of-hospital cardiac arrest. Resuscitation 2013;84:915-20.

37. Stiell IG, Walker RG, Nesbitt LP, et al. BIPHASIC Trial: a randomized comparison of fixed lower versus escalating higher energy levels for defibrillation in out-of-hospital cardiac arrest. Circulation 2007;115:1511-7.

38. Koster RW, Walker RG, Chapman FW. Recurrent ventricular fibrillation during advanced life support care of patients with prehospital cardiac arrest. Resuscitation 2008;78:252-7.

39. Cheskes S, Schmicker RH, Verbeek PR, et al. The impact of peri-shock pause on survival from out-of-hospital shockable cardiac arrest during the Resuscitation Outcomes Consortium PRIMED trial. Resuscitation 2014;85:336-42.

40. Sell RE, Sarno R, Lawrence B, et al. Minimizing pre- and postdefibrillation pauses increases the likelihood of return of spontaneous circulation (ROSC). Resuscitation 2010;81:822-5.

41. Thel MC, Armstrong AL, McNulty SE, Califf RM, O'Connor CM. Randomised trial of magnesium in in-hospital cardiac arrest. Duke Internal Medicine Housestaff. Lancet 1997;350:1272-6. 42. Shin TG, Jo IJ, Song HG, Sim MS, Song KJ. Improving survival rate of patients with in-hospital cardiac arrest: five years of experience in a single center in Korea. J Korean Med Sci 2012; 27:146-52.

43. Kwak HJ, Yun I, Kim SH, et al. The extended rapid response system: 1-year experience in a university hospital. J Korean Med Sci 2014;29:423-30.

44. Crowe $\mathrm{C}$, Bobrow BJ, Vadeboncoeur TF, et al. Measuring and improving cardiopulmonary resuscitation quality inside the emergency department. Resuscitation 2015;93:8-13.

45. Hamrick JL, Hamrick JT, Lee JK, Lee BH, Koehler RC, Shaffner DH. Efficacy of chest compressions directed by end-tidal $\mathrm{CO} 2$ feedback in a pediatric resuscitation model of basic life support. J Am Heart Assoc 2014;3:e000450.

46. Genbrugge $C$, Meex I, Boer W, et al. Increase in cerebral oxygenation during advanced life support in out-of-hospital patients is associated with return of spontaneous circulation. Crit Care 2015;19:112.

47. Soar J, Callaway CW, Aibiki M, et al. Part 4. Advanced life support: 2015 International Consensus on Cardiopulmonary Resuscitation and Emergency Cardiovascular Care Science with Treatment Recommendations. Resuscitation 2015;95:e71-120.

48. Callaham M, Madsen CD, Barton CW, Saunders CE, Pointer J. A randomized clinical trial of high-dose epinephrine and norepinephrine vs standard-dose epinephrine in prehospital cardiac arrest. JAMA 1992;268:2667-72.

49. Helm C, Gillett M. Adrenaline in cardiac arrest: prefilled syringes are faster. Emerg Med Australas 2015;27:312-6.

50. Moreira ME, Hernandez $C$, Stevens AD, et al. Color-coded prefilled medication syringes decrease time to delivery and dosing error in simulated emergency department pediatric resuscitations. Ann Emerg Med 2015;66:97-106.e3.

51. Neumar RW, Otto CW, Link MS, et al. Part 8. Adult advanced cardiovascular life support: 2010 American Heart Association Guidelines for Cardiopulmonary Resuscitation and Emergency Cardiovascular Care. Circulation 2010;122(18 Suppl 3):S72967.

52. Hess EP, Russell JK, Liu PY, White RD. A high peak current 150-J fixed-energy defibrillation protocol treats recurrent ventricular fibrillation (VF) as effectively as initial VF. Resuscitation 2008;79:28-33.

53. Kudenchuk PJ, Cobb LA, Copass MK, et al. Amiodarone for resuscitation after out-of-hospital cardiac arrest due to ventricular fibrillation. N Engl J Med 1999;341:871-8.

54. Dorian P, Cass D, Schwartz B, Cooper R, Gelaznikas R, Barr A. Amiodarone as compared with lidocaine for shock-resistant ventricular fibrillation. N Engl J Med 2002;346:884-90.

55. Soar J, Nolan JP, Bottiger BW, et al. European Resuscitation 
Council Guidelines for Resuscitation 2015. Section 3: adult advanced life support. Resuscitation 2015;95:100-47.

56. Herlitz J, Ekstrom L, Wennerblom B, et al. Lidocaine in out-ofhospital ventricular fibrillation: does it improve survival? Resuscitation 1997;33:199-205.

57. Harrison EE. Lidocaine in prehospital countershock refractory ventricular fibrillation. Ann Emerg Med 1981;10:420-3.

58. Spindelboeck W, Schindler O, Moser A, et al. Increasing arterial oxygen partial pressure during cardiopulmonary resuscitation is associated with improved rates of hospital admission. Resuscitation 2013;84:770-5.

59. Breitkreutz R, Price $S$, Steiger HV, et al. Focused echocardio- graphic evaluation in life support and peri-resuscitation of emergency patients: a prospective trial. Resuscitation 2010;81: 1527-33.

60. Chou HC, Chong KM, Sim SS, et al. Real-time tracheal ultrasonography for confirmation of endotracheal tube placement during cardiopulmonary resuscitation. Resuscitation 2013;84:170812.

61. Maekawa K, Tanno K, Hase M, Mori K, Asai Y. Extracorporeal cardiopulmonary resuscitation for patients with out-of-hospital cardiac arrest of cardiac origin: a propensity-matched study and predictor analysis. Crit Care Med 2013;41:1186-96. 